\title{
RELACIÓN ENTRE EL VOLUMEN QUIRÚRGICO Y LOS RESULTADOS POSTOPERATORIOS EN ESOFAGUECTOMÍA Y PANCREATECTOMÍA POR CÁNCER*
}

\author{
Drs. Attila Csendes J. ${ }^{1}$, Marcelo Zamorano D. ${ }^{1}$, Jaime Matus R. ${ }^{1}$, Galo Cárdenas S. ${ }^{1}$ \\ ${ }^{1}$ Departamento de Cirugía, Hospital Clínico Universidad de Chile. \\ Chile.
}

\begin{abstract}
Inverse relationship between intervention volume and complications of esophagectomy and pancreatectomy. A review

Background: The prevalence of pancreatic cancer increased in Chile in the last decade, while that of esophageal cancer is decreasing. Both diseases are a surgical challenge due to their high mortality and rate of complications. Aim: To analyze the relationship between intervention volume and surgical complications. Material and Methods: We reviewed 19 papers analyzing the relationship between the number of interventions performed in a determinate hospital (yearly hospital volume), the number of interventions performed by each surgeon (yearly surgical volume) and the rates of mortality and complications of both conditions. Results: There is a clear inverse relationship between volumes and complications. There is also a higher five years survival in high volume hospitals. Conclusions: Intervention volume is inversely associated with rates of complications.
\end{abstract}

Key words: Surgical volume, complications, pancreatectomy, esophagectomy.

\section{Resumen}

En la última década el cáncer pancreático ha experimentado un alza sostenida en su prevalencia en Chile, mientras que el cáncer esofágico ha mostrado una tendencia hacia su descenso. Ambas patologías son de gran complejidad, representando un gran desafío al grupo quirúrgico por su alta morbilidad y mortalidad. En el presente estudio se revisaron 19 artículos que evalúan la relación entre números de operaciones efectuadas por año en un Hospital (volumen hospitalario/año) y el número de cirugías por cirujanos (volumen quirúrgico/año) y la morbimortalidad de ambas patologías. Los resultados demuestran claramente una relación inversa entre mayor volumen y menor morbimortalidad tanto para la esofaguectomía total como la pancreatoduodenectomía. También se demostró una mayor sobrevida a 5 años en los hospitales de alto volumen.

*Recibido el 24 de septiembre de 2014 y aceptado para publicación el 12 de noviembre de 2014.

Los autores no refieren conflictos de interés.

Correspondencia: Dr. Attila Csendes J. acsendes@hcuch.cl 
Estos hallazgos pueden ser de gran utilidad para el Ministerio de Salud, si considerara estos resultados. La concentración de ambas patologías en unos pocos centros quirúrgicos lograría obtener una mejor evolución postoperatoria y una mayor sobrevida, junto con un importante ahorro económico al controlar mejor los gastos en que se incurre al atender a estos pacientes de alta complejidad.

Palabras clave: Esofaguectomía, pancreatoduodenectomía, mortalidad, morbilidad, sobrevida, volumen quirúrgico.

\section{Introducción}

Desde 1979 se ha publicado la relación inversamente proporcional entre el volumen quirúrgico y la mortalidad ${ }^{1}$. Esto se acentúa frente a patologías poco frecuentes y de resoluciones quirúrgicas técnicamente complejas. Dos entidades que representan este problema son, por un lado el cáncer esofágico, el que se encuentra entre las 10 principales causas de mortalidad por cáncer a nivel mundial, con un pronóstico extremadamente ominoso, constituyendo la esofaguectomía una parte del tratamiento multimodal de esta patología ${ }^{2}$, siendo realizada comúnmente en pacientes de alto riesgo y añosos, con múltiples comorbilidades cardiopulmonares y nutricionales; el éxito de la esofaguectomía depende de la presencia de un equipo quirúrgico experimentado que trabaje en el contexto de un programa multidisciplinario bien estructurado. Por otro lado se encuentran los tumores periampulares, siendo el más frecuente el cáncer pancreático, que ocupa el $13^{\text {vo }}$ lugar de incidencia y el $8^{\text {vo }}$ en mortalidad a nivel mundial ${ }^{3}$. Según el Survelliance Epidemiology and End Results de USA (SEER) ${ }^{4}$, la tasa de incidencia es de 12.1/100.000, con pacientes de similares características que en el cáncer de esófago, es decir, pacientes añosos con comorbilidades y generalmente con enfermedad metastásica (53\%) al momento del diagnóstico, siendo sólo un $8 \%$ considerado con compromiso local exclusivo ${ }^{3-5}$.

En la actualidad el tratamiento de elección del carcinoma pancreático es la cirugía $a^{4,6}$, la cual se realiza en muchos hospitales alrededor del mundo, con una mortalidad perioperatoria que varía entre 5 y $12 \%$, y una tasa de complicaciones del 15 a $50 \%$ según el centro hospitalario, con una relación inversamente proporcional entre el volumen hospitalario de casos y la mortalidad y morbilidad perioperatoria, pero sin variaciones en la mortalidad a largo plazo según el centro ${ }^{5-8}$.

Las series de casos realizadas en centros académicos sugieren la posibilidad de realizar este procedimiento con relativa seguridad y con una mortalidad reportada de 4-6\%. Sin embargo, estudios poblacionales de gran volumen sugieren una mortalidad considerablemente más alta, particularmente en hospitales con baja experiencia en este procedimiento ${ }^{3}$, demostrando de esta manera la relación inversamente proporcional entre la mortalidad operatoria y el volumen de casos por hospital.

El objetivo de esta revisión es analizar la relación entre el volumen quirúrgico y los resultados operatorios en la esofaguectomía y la pancreato duodenectomía, dando una visión crítica al estado actual de esta situación en nuestro país.

\section{Esofaguectomía}

\section{Relación entre el volumen de casos por hospital y mortalidad postoperatoria}

En el año 2002 Birkmeyer y colaboradores ${ }^{9,10}$, publican un estudio en el que analizan la mortalidad en la esofaguectomía (entre otros procedimientos), enrolando a 6.337 pacientes sometidos a esta operación en 1.575 hospitales (Tabla 1), reportando una mortalidad operatoria (definida como mortalidad intrahospitalaria o dentro de los primeros 30 días postesofaguectomía) significativamente mayor en los hospitales de bajo volumen (menos de 2 procedimientos al año) comparados con los hospitales de alto volumen (más de 19 procedimientos al año), $23,1 \%$ vs $8,1 \%$ respectivamente. En otro estudio publicado por Begg y colaboradores ${ }^{9,11}$ en 1998, reportan una mortalidad postoperatoria a 30 días de $17,3 \%$ y $3,4 \%$ entre los hospitales de bajo volumen (menos de 5 esofaguectomías al año) y los hospitales de alto volumen respectivamente (más de 11 esofaguectomías al año).

Por otra parte en el 2002, Gillison y colabores encuentran una mortalidad postoperatoria a 30 días de aproximadamente $10 \%$, sin apreciar diferencias significativas entre los hospitales que realizan más de 10 esofaguectomías al año y los que realizan menos de 10 esofaguectomías al año. Sin embargo, los autores notan una mortalidad significativamente más alta (superior al 20\%) en los hospitales que realizan menos de 2 operaciones al año $0^{9,12}$.

\section{Relación entre el volumen de casos por hospital y las complicaciones postoperatorias}

Reavis y cols. publican en el 2008 un estudio en el que analizan el impacto del volumen de esofaguectomías realizadas por hospital en los resultados postoperatorios, utilizando la base de datos del Uni- 
Tabla 1. Impacto del volumen hospitalario en la mortalidad postoperatoria en esofaguectomía

\begin{tabular}{|c|c|c|c|c|c|c|}
\hline \multirow[t]{2}{*}{ Autor } & \multirow{2}{*}{$\begin{array}{c}\text { n de } \\
\text { pacientes }\end{array}$} & \multicolumn{3}{|c|}{ Mortalidad (\%) ajustada por volumen hospitalario } & \multirow[t]{2}{*}{$\mathbf{p}$} & \multirow[t]{2}{*}{ Mortalidad } \\
\hline & & $\begin{array}{c}\text { Bajo } \\
\text { (procedimientos } \\
\text { por año) }\end{array}$ & $\begin{array}{c}\text { Medio } \\
\text { (procedimientos } \\
\text { por año) }\end{array}$ & $\begin{array}{c}\text { Alto } \\
\text { (procedimientos } \\
\text { por año) }\end{array}$ & & \\
\hline Begg et al. & 503 & $\begin{array}{l}17,3 \\
(\leq 5)\end{array}$ & $\begin{array}{c}3,9 \\
(6-10)\end{array}$ & $\begin{array}{c}3,4 \\
\geq 11\end{array}$ & $<0,001$ & 30 días \\
\hline Patti et al. & 1.561 & $\begin{array}{l}16,1 \\
(\leq 6)\end{array}$ & - & $\begin{array}{l}4,8 \\
\geq 6\end{array}$ & $<0,0001$ & Operatoria \\
\hline Gordon et al. & 518 & $\begin{array}{l}14,8-15,1 \\
\quad(<20)\end{array}$ & $\begin{array}{c}9,3 \\
(21-50)\end{array}$ & $\begin{array}{c}3,1 \\
\geq 50\end{array}$ & $<0,001$ & Intra-hospitalaria \\
\hline Swisher et al. & 340 & $\begin{array}{l}12,2 \\
(<5)\end{array}$ & - & $\begin{array}{c}3 \\
\geq 5\end{array}$ & 0,004 & Operatoria \\
\hline Van Lanschot & 1.792 & $\begin{array}{l}12,1 \\
(\leq 10)\end{array}$ & $\begin{array}{c}7,5 \\
(11-20)\end{array}$ & $\begin{array}{c}4,9 \\
\geq 20\end{array}$ & $<0,001$ & Hospitalaria \\
\hline Dimick et al. & 1.136 & $\begin{array}{c}16 \\
(\leq 3)\end{array}$ & $\begin{array}{c}12,7 \\
(4-15)\end{array}$ & $\begin{array}{l}2,7 \\
\geq 15\end{array}$ & $<0,001$ & Intra-hospitalaria \\
\hline Kuo et al. & 1.193 & $\begin{array}{l}9,2 \\
(\leq 6)\end{array}$ & - & $\begin{array}{l}2,5 \\
\geq 6\end{array}$ & $<0,001$ & Intra-hospitalaria \\
\hline Gillison et al. & 1.125 & $\begin{array}{c}9,8 \\
(\leq 10)\end{array}$ & - & $\begin{array}{l}10,4 \\
\geq 10\end{array}$ & NS & 30 días \\
\hline Birkmeyer et al. & 6.337 & $\begin{array}{l}20,3 \\
(<5)\end{array}$ & $\begin{array}{c}14 \\
(5-19)\end{array}$ & $\begin{array}{c}8,1 \\
>19\end{array}$ & $<0,001$ & $\begin{array}{c}\text { Hospitalaria y a } \\
30 \text { días }\end{array}$ \\
\hline Urbach et al. & 613 & $\begin{array}{l}18,6 \\
(<3)\end{array}$ & $\begin{array}{c}12,4 \\
(3-18)\end{array}$ & $\begin{array}{l}10,2 \\
>19\end{array}$ & $<0,03$ & 30 días \\
\hline McCulloch et al. & 955 & $\begin{array}{l}16,7 \\
(<11)\end{array}$ & $\begin{array}{c}12,7 \\
(11-20)\end{array}$ & $\begin{array}{r}10,3 \\
>20\end{array}$ & NS & 30 días \\
\hline Wenner et al. & 1.429 & $\begin{array}{l}10,4 \\
(<5)\end{array}$ & $\begin{array}{c}6,3 \\
(5-15)\end{array}$ & $\begin{array}{c}3,5 \\
>15\end{array}$ & 0,02 & Hospitalaria \\
\hline
\end{tabular}

Adaptado de Chang A, Birkmeyer J. The volume-performance relationship in esophagectomy. Thorac Surg Clin. 2006;16:87-94.

versity Health System Consortium. Los objetivos de dicho estudio fueron: estancia hospitalaria, reingreso hospitalario en los primeros 30 días, morbilidad y mortalidad observada y esperada, entre los hospitales con alto (más de 12), medio (6-11) y bajo (menos de 5 esofaguectomías al año) volumen. Encontraron que los hospitales con alto volumen comparados con los hospitales de bajo volumen tienen menor estancia intrahospitalaria (14,1 vs 17,2 días), menos complicaciones $(51,1 \%$ vs $56,5 \%)$ y una menor mortalidad intrahospitalaria $(2,5 \%$ vs $5,6 \%)$ resultando todas estas estadísticamente significativas, por lo que concluyen que se logran mejores resultados postoperatorios y menor mortalidad en los centros hospitalarios que realizan más de 12 esofaguectomías al año ${ }^{13}$.

En 1998, Patti y cols. publican un trabajo en el que analizan las tasas de incidencia de infección del sitio quirúrgico y de sangrado postoperatorio en esofaguectomía sin encontrar diferencia estadísticamente significativa entre los hospitales de alto volumen con los de bajo volumen. Sin embargo, la mortalidad en los pacientes que presentaron infección postoperatoria fue mayor en los hospitales de bajo volumen encontrándose diferencia estadísticamente significativa $(p=0,008)$.

\section{Impacto del volumen y la supervivencia}

Existe una clara relación entre el volumen de esofaguectomías realizadas por hospital y la supervivencia reportada en los pacientes, tal como lo demuestra Wenner en 2005 al reportar una supervivencia a 5 años del $22 \%$ en los hospitales que realizan más de 15 procedimientos al año, frente al 17\% de supervivencia lograda en los centros hospitalarios que realizan menos de 5 procedimientos al año. 


\section{Pancreatoduodenectomía}

\section{Relación entre el volumen de casos por hospital y la mortalidad postoperatoria en cáncer de páncreas}

En 1998 Berg y cols. ${ }^{6}$, realizaron un análisis retrospectivo acerca de la mortalidad operatoria ( $<30$ días) y el impacto de volumen hospitalario en cirugía mayor para cáncer en los Estados Unidos usando la base de datos del SEER (Surveillance, Epidemiology and End Results) dentro de los cuales se incluyó la Pancreatectomía con un valor de 12,9\% para los centros con bajo volumen ( $\mathrm{n}=<5$ cirugías/ año); 7,7\% en centros con alto volumen $(\mathrm{n}=6-10$ cirugías/año) y de 5,8\% para los centros con muy alto volumen ( $>10$ Pancreatectomías por año), demostrando una tendencia de disminución de la mortalidad con el aumento de volumen hospitalario $(\mathrm{p}=0,004)$.

Birkmeyer y cols. ${ }^{7}$ en 1999, presentaron los resultados de 7.229 pacientes sometidos a pancreatoduodenectomía (operación de Whipple) en los Estados Unidos categorizándolos en 5 grupos en función del volumen: muy bajo $(<1$ casos/año), bajo (1-2 casos/año ), medio (3-5 casos/año) y alto (5-14) y muy alto (> 15 casos por año). Reportaron tasas de mortalidad en hospitales de bajo y muy bajo volumen de 3 a 4 veces más alta que en los hospitales de alto volumen ( $12 \%$ y $16 \%$, respectivamente), frente al $2 \%(\mathrm{p}<0,001)$, cuando se realizaban más de 15 casos por año. Existieron 10 hospitales con volúmenes más altos de la nación, que tenían tasas de mortalidad más bajas que los centros de alto volumen restantes: $2,1 \%$ vs $6,2 \%$, $(\mathrm{p}<0,01)$ (Tabla 2 y Figura 1).

El mismo grupo el año $2002^{8}$, analizó el volumen hospitalario y la mortalidad quirúrgica en los
Estados Unidos, utilizando dos bases de datos, demostrando que la tasa de mortalidad en resecciones pancreáticas para los hospitales con bajo volumen fue de 14 a $16 \%$, más alta que en los hospitales de muy alto volumen $(3,8 \%)$, con una disminución progresiva de la mortalidad según el número de casos por año, con una relación inversamente proporcional como lo demuestra la Tabla 3.

Balzano T. y cols. ${ }^{14}$, incluyeron a 1.576 pacientes que se sometieron a pancreatoduodenectomía los que fueron asignados a cuatro grupos de volumen:

Tabla 2. Mortalidad según volumen en pancreatoduodenectomías (Birkmeyer, 1999)

\begin{tabular}{|lcc|}
\hline Volumen & Casos/año & Mortalidad en \% \\
\hline Muy bajo & $<1$ & 16 \\
\hline Bajo & $1-2$ & 12 \\
\hline Medio & $3-5$ & 11,0 \\
\hline Alto & $>5-14$ & 6,2 \\
\hline Muy alto & $>15$ & 2,1 \\
\hline
\end{tabular}

Tabla 3. Impacto del número de operaciones anuales y mortalidad postoperatoria en pacientes con carcinoma pancreático (Birkmeyer, 2002)

\begin{tabular}{|lcc|}
\hline Volumen & Casos/año & Mortalidad en \% \\
\hline Muy bajo & $<1$ & 16,3 \\
Bajo & $1-2$ & 14,0 \\
Medio & $3-5$ & 11,0 \\
Alto & $6-16$ & 7,2 \\
Muy alto & $>16$ & 3,8 \\
\hline
\end{tabular}

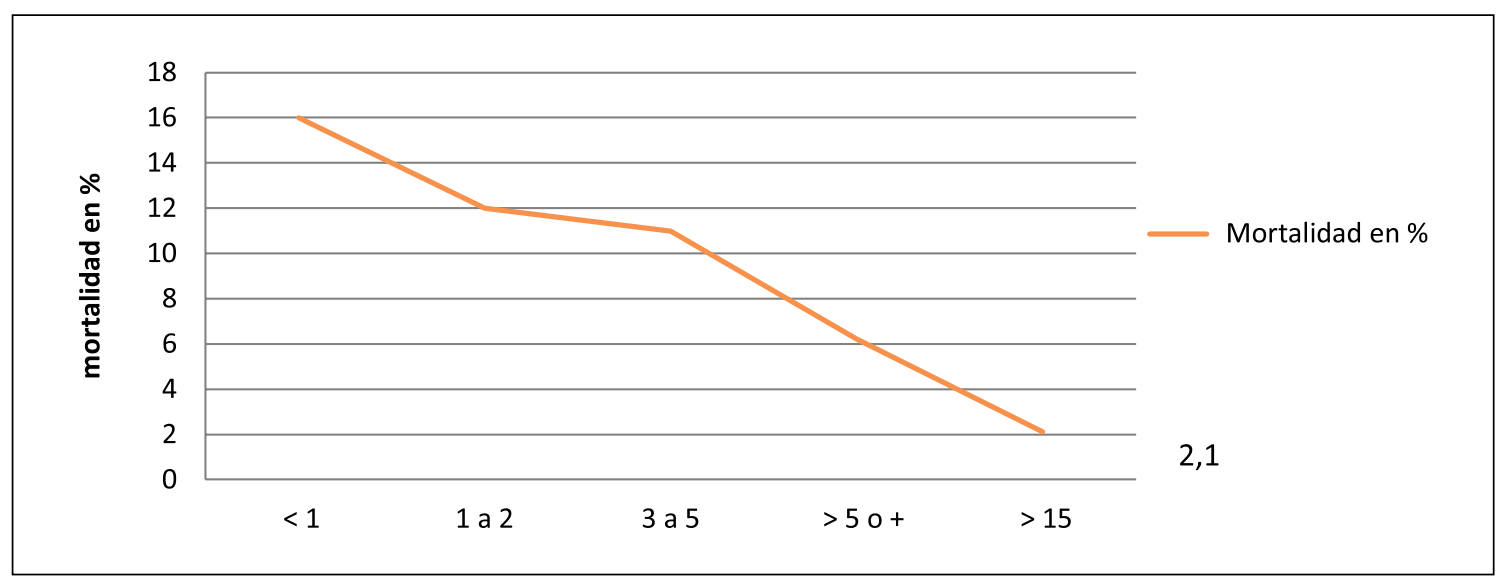

Figura 1. Mortalidad según volumen en pancreaduodenectomía (Birkmeyer). 
1) Bajo (<5 x año); 2) Medio (6-13 x año); 3) Alto volumen (14-50 x año); 4) Muy alto volumen (>50 x año). La mortalidad global fue del 8,1\%. El aumento de volumen hospitalario se asoció con una tasa de mortalidad significativamente menor: $12,4 \%-7,8 \%-5,9 \%$ y un $2,6 \%$ respectivamente para cada uno de los grupos (Figura 2). La duración de la estancia postoperatoria también se redujo en los hospitales de alto volumen $(\mathrm{p}<0,001)$.

\section{Impacto del volumen por cirujano en la mortalidad hospitalaria}

La presencia de tasas de mortalidad operatoria menores en los centros de alto volumen después de la resección pancreática ${ }^{3-8,15,16}$ ha planteado también el impacto del volumen cirujano en la mortalidad.

El volumen cirujano es definido como la cantidad de procedimientos (resecciones pancreáticas) realizadas por un cirujano en un período de tiempo determinado (en este caso es de un año) ${ }^{17}$.

Eppsteiner y cols. ${ }^{17}$, analizaron 3.581 resecciones pancreáticas usando la base de datos del NIS ( $\mathrm{Na}$ tionwide Inpatient Sample) identificando a los cirujanos que las realizaron y dividiéndolos en dos grupos:

- Alto volumen cirujano $(\mathrm{AV})=>0=5$ operaciones/año.

- Bajo volumen cirujano (BV) $=<5$ operaciones/ año.

Identificaron 134 cirujanos de alto volumen y 1.450 cirujanos de bajo volumen, los cuales realizaron en total 3.581 resecciones pancreáticas en 742 hospitales en 12 Estados en un período de 8 años. La operación más realizada fue la pancreatoduodenectomía $(71,7 \%)$ y en segundo lugar la pancreatectomía distal $(28,3 \%)$. Los cirujanos de alto volumen completaron más pancreatoduodenectomía que pancreatectomías distales $(79-21 \%$ respectivamente). Los pacientes que fueron sometidos a estos procedimientos por cirujanos de AV tuvieron una mortalidad ajustada de $2,4 \%$, más baja en comparación con los cirujanos $\mathrm{BV}$ con una tasa del $6,4 \%$, $(\mathrm{p}<0,0001)^{17}$, demostrando así la existencia de una relación inversamente proporcional de relación volumen cirujano/mortalidad hospitalaria: $>$ volumen $\mathrm{x}$ cirujano $<$ mortalidad.

\section{Relación entre el volumen de casos por hospital y las complicaciones postoperatorias}

Dimick y cols., publicaron un estudio en el 2003 acerca de la variación de las complicaciones postoperatorias luego de cirugía de alto riego en los Estados Unidos, utilizando la base de datos del NIS (Nationwide Inpatient Sample); se incluyeron tres operaciones (esofaguectomía $(\mathrm{n}=1.226)$, la pancreatectomía $(\mathrm{n}=4.789)$, y la reparación de aneurisma de aorta abdominal $(\mathrm{n}=11.863)$. Las complicaciones evaluadas incluyeron aspiración, complicaciones cardíacas, neumonía, insuficiencia pulmonar, insuficiencia renal, y otros ${ }^{18}$.

La ocurrencia de una o más complicaciones fue asociada con un incremento del riesgo de mortalidad. Los pacientes con una o más complicaciones después de la resección pancreática tenían una mortalidad del 18,8\% frente a sólo el 5,2\% para aquellos sin complicaciones $(\mathrm{p}<0,001)$.

En el análisis considerando el volumen hospitalario se evidenció una variación de pacientes que presentaron una complicación postoperatoria, siendo del $6 \%$ en los centros de alto volumen vs $12 \%$ en los de bajo volumen $(\mathrm{p}<0,001)$. Además la resección pancreática dentro de los centros de alto volumen presentó menor riesgo de desarrollar complicaciones severas como aspiración, falla respiratoria, fallo renal y sepsis; en general los centros de alto volumen tienen tasas más bajas de una o más complicaciones después de la resección pancreática (OR: 0,71 , IC $95 \%, 0,57$ a $0,83, \mathrm{p}=0,002)^{18}$.

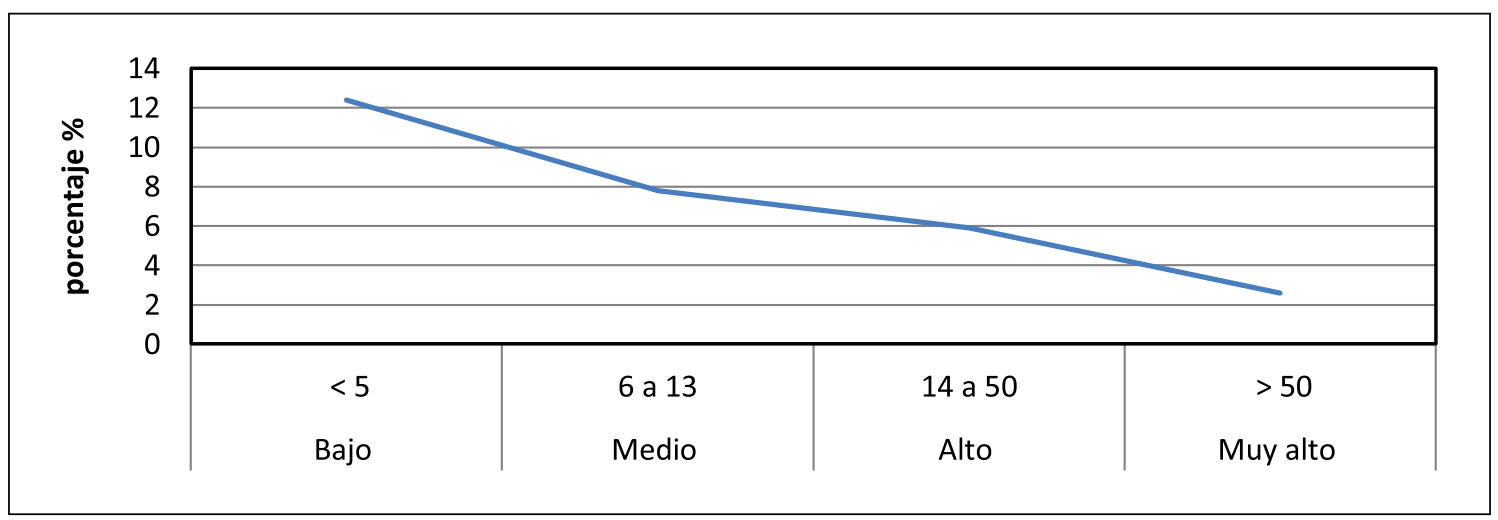

Figura 2. Mortalidad según volumen en pancreaduodenectomía (Balzano). 
Las operaciones de alto riesgo tienen una menor tasa de complicaciones postoperatorias cuando se realiza en hospitales de alto volumen ${ }^{8,15,18}$. Los resultados globales de la pancreatectomía se muestran en la Tabla 4.

\section{Impacto del volumen quirúrgico en la sobrevida}

En la parte occidental de los Países Bajos, la red profesional de los cirujanos oncólogos acordó centralizar todas las cirugías de páncreas desde 2006 en dos hospitales de alto volumen. La tasa de supervivencia a los 2 años se incrementó después de la centralización del 39\% al 55\% $(\mathrm{p}=0,09)$ para todos los pacientes que tuvieron una resección pancreática por cáncer de páncreas ${ }^{15}$. Después de ajustar por edad, localización tumoral, estadio, histopatología y tratamiento adyuvante, el último período se asoció significativamente con una mejor supervivencia (hazard ratio [HR] 0,50; intervalo de confianza del 95\% [IC 95\%] 0,34-0,73) $)^{15}$.

Se ha demostrado que la presencia de mayores tasas de supervivencia a largo plazo se asocian con resecciones viscerales complejas como tratamiento para los cánceres, realizadas en los centros de alto volumen $^{14,15-19}$.

Fong Youman y cols., estudiaron a los pacientes que fueron sometidos a pancreatectomía o hepatectomía debido a cáncer en los años 1995 y 1996 iden- tificados en la base de datos nacional de Medicare $^{19}$, analizando la supervivencia a largo plazo confirmada en el año 2001, obteniendo de esta manera una sobrevida real a 5 años. En el período de estudio, hubo 2.592 pancreatectomías. La comparación de log-Rank demostró una sobrevida superior en los pacientes resecados en los centros de alto volumen vs los centros de bajo volumen (pancreatectomías: $\mathrm{p}=0,001)^{19}$, similar a los reportes de otras series con una sobrevida a cinco años superior al $5 \%{ }^{16}$, por lo cual queda demostrado que la especialización y centralización a centros de alto volumen hospitalario $\mathrm{y}$ de volumen cirujano genera un gran impacto en la mortalidad a mediano y largo plazo $0^{17-19}$.

En Chile, Los datos oficiales del Ministerio de Salud muestran (Tabla 5) que la mortalidad anual es casi equivalente a la prevalencia de estos 2 cánceres, debido a su altísima letalidad antes de 1 año de su diagnóstico. El cáncer de esófago ha ido disminuyendo en la última década en un $9 \%$ y el cáncer de páncreas ha experimentado un continuo ascenso de un 37\%. Llama la atención que el año 2011 sólo se efectuaron 42 esofaguectomías por cáncer de esófago en todo el país, así como 102 pancreatectomías, de las cuales 69 fueron pancreatoduodenectomías, con una mortalidad de $8,7 \%$. Estas cifras claramente refuerzan la necesidad de concentrar la atención de estas patologías en 2 a 3 hospitales en todo Chile.

Tabla 4. Relación entre el volumen hospitalario y los resultados postoperatorios en pancreatectomía

\begin{tabular}{|c|c|c|c|c|c|c|c|c|c|c|c|c|}
\hline \multirow[b]{2}{*}{ Autores } & \multirow[b]{2}{*}{$\mathbf{n}$} & \multicolumn{4}{|c|}{$\begin{array}{c}\text { Volumen } \\
\text { Casos por año }\end{array}$} & \multicolumn{4}{|c|}{$\begin{array}{c}\text { Mortalidad (\%) } \\
\text { Por volumen: hospitalario o cirujano }\end{array}$} & \multicolumn{2}{|c|}{$\begin{array}{l}\text { Complicaciones } \\
\text { Según el centro }\end{array}$} & \multirow[t]{2}{*}{$\begin{array}{c}\text { Valor } \\
\mathbf{p}\end{array}$} \\
\hline & & Bajo & Medio & Alto & $\begin{array}{l}\text { Muy } \\
\text { alto }\end{array}$ & Bajo & Medio & Alto & $\begin{array}{l}\text { Muy } \\
\text { alto }\end{array}$ & Alto & Bajo & \\
\hline $\begin{array}{l}\text { Begg C, } \\
\text { et al. } 2010\end{array}$ & 5.013 & $<5$ & - & $6-10$ & $>10$ & 12,9 & - & 7,7 & 5,8 & - & - & $<0,001$ \\
\hline $\begin{array}{l}\text { Birkmeyer JD, } \\
\text { et al. } 2002\end{array}$ & 474.108 & $1-2$ & $3-5$ & $6-16$ & $>16$ & 14,0 & 11,0 & 7,2 & 3,8 & - & - & $<0,001$ \\
\hline $\begin{array}{l}\text { Birkmeyer JD, } \\
\text { et al. } 1999\end{array}$ & 7.229 & $1-2$ & $3-5$ & $>5$ & $>15$ & 12 & 11 & 6,2 & 2,1 & - & - & $<0,001$ \\
\hline $\begin{array}{l}\text { Balzano G, } \\
\text { et al. } 2008\end{array}$ & 1.576 & $<5$ & $6-13$ & $14-50$ & $>50$ & 12,4 & 7,8 & 5,9 & 2,6 & - & - & $<0,001$ \\
\hline $\begin{array}{l}\text { Eppsteiner, } \\
\text { et al. } 2009\end{array}$ & 1.584 & $<5$ & - & $>5$ & - & 6,4 & - & 2,4 & - & - & - & $<0,0001$ \\
\hline $\begin{array}{l}\text { Gooiker GA, } \\
\text { et al. } 2011\end{array}$ & 249 & - & - & - & - & 36 & - & 55 & - & & & $<0,001$ \\
\hline $\begin{array}{l}\text { Dimick JB, } \\
\text { et al. } 2003\end{array}$ & 4.789 & - & - & - & - & 18,8 & - & 5,2 & - & 6 & 12 & $\begin{array}{l}<0,001 \mathrm{y} \\
<0,002\end{array}$ \\
\hline $\begin{array}{l}\text { Youman, } \\
\text { et al. } 2005\end{array}$ & 2.592 & - & - & - & - & 8 & & 2 & & & & $<001$ \\
\hline
\end{tabular}


Tabla 5. Situación del cáncer de esófago y de páncreas en Chile. Años 2000, 2006 y 2011

\begin{tabular}{|lcc|}
\hline & $\begin{array}{c}\text { Cáncer } \\
\text { esófago }\end{array}$ & $\begin{array}{c}\text { Cáncer } \\
\text { páncreas }\end{array}$ \\
Año 2000 & 744 & 695 \\
Total fallecidos & 4,8 & 4,5 \\
Tasa & & \\
Año 2006 & 726 & 926 \\
Total fallecidos & 4,4 & 5,6 \\
Tasa & & \\
Año 2011 & 683 & 1108 \\
Total fallecidos & 4,0 & 6,4 \\
Tasa & $-9 \%$ & $+37 \%$ \\
Diferencia 2000-2011 & 42 & 69 \\
Total resecados & & \\
2011 & $4(9,5 \%)$ & $6(8,7 \%)$ \\
Mortalidad op. & & \\
\hline
\end{tabular}

Tasa: número de fallecidos por 100.000 habitantes.

\section{Conclusión}

Los datos analizados en el presente estudio sugieren fuertemente que hay una correlación inversa entre el mayor número de pacientes operados por cáncer esofágico o pancreático, realizando esofaguectomía total o pancreatoduodenectomía respectivamente, y la morbimortalidad postoperatoria. Estas cifras también sugieren que el Ministerio de Salud de Chile debiera tomar en cuenta estos resultados y concentrar estas patologías en 2 a 3 centros nacionales, con lo que, por una parte se aumentaría la experiencia de los grupos dedicados a estas patologías, y por otra parte, que es la más importante, la evolución de estos pacientes graves sometidos a una cirugía altamente compleja sería mucho más favorable, con una pronta recuperación postoperatoria, con menor morbilidad y mortalidad. Finalmente, para el país, esto también representaría un fuerte ahorro económico, al disminuir los importantes gastos en que se incurre al tratar estos pacientes.

\section{Referencias}

1. Luft HS, Bunker JP, Enthoven AC. Should operations be regionalized? The empirical relation between surgical volume and mortality. N Engl J Med. 1979;301:1364-9.

2. Jemal A, Murray T, Ward E, Samuels A, Tiwari RC, Ghafoor A, et al. Cancer statistics 2005. CA Cancer
J Clin. 2005;55:10-30.

3. Globocan 2008. Organización Mundial de la Salud (OMS).

4. Survilliance Epidemiology \& End Results (SEER) of the United States of America.

5. Mayo SC, Gilson MM, Herman JM, Cameron JL, Nathan H, Edil BH, et al. Management of patient with adenocarcinoma: national trends in patients selection, operative management, and use of adjuvant therapy. J Am Coll Surg. 2012;214:33-5.

6. Begg C, Cramer L, Hoskins W, Brennan M. Impact of hospital volume on operative mortality for major cancer surgery. JAMA 1998;280:1747-51.

7. Birkmeyer JD, Finlayson SR, Tosteson AN, Sharp SM, Warshaw AL, Fisher ES. Effect of hospital volumen on in-hospital volumen with pancreaticoduodenectomy; Surgery 1999;125;250-6.

8. Birkmeyer JD, Siewers AE, Finlayson EV, Stukel TA, Lucas FL, Batista I, et al. Hospital Volume And Surgical Mortality In The United States. N Engl J Med. 2002:346;1128-37.

9. Chang A, Birkmeyer JD. The volume-performance relationship in esophagectomy. Thorac Surg Clin. 2006;16:87-94.

10. Gillison EW, Powell J, Mc Conkey CC, Spychal RT. Surgical Workload and outcome after resection for carcinoma of the oesophagus and cardia. Br J Surg. 2002;89:344-8.

11. Reavis KM, Smith BR, Hinojosa MW, Nguyen NT. Outcomes of esophagectomy at academic centers: An association between volume and outcome. Am Surg. 2008;74:939-43.

12. Balzano G, Zerbi A, Capretti G, Rocchetti S, Capitanio V, Di Carlo V. Effect of hospital volume on outcome of pancreaticoduodenectomy in Italy; Brit J Surg. 2008;95:357-62.

13. Gooiker GA, Van der Geest LG, Wouters MW, Vonk M, Karsten TM, Tollenaar RA, et al. Quality improvement of pancreatic surgery by centralization in the western part of the Netherlands. Ann Surg Oncol. 2011;18:1821-9.

14. Birkmeyer JD, Sun Y, Wong SL, Stukel TA. Hospital volume and late survival after cancer surgery. Ann Surg. 2007;245:777-83.

15. Eppsteiner RW, Csikesz NG, McPhee JT, Tseng JF, Shah SA. Surgeon volume impacts hospital mortality for pancreatic resection; Ann Surg. 2009;249:635-40.

16. Dimick JB, Pronovost PJ, Cowan JA Jr, Lipsett PA, Stanley JC, Upchurch GR Jr. Variation in postoperative complication rates after high-risk surgery in the United States. Surgery 2003;134:534-40.

17. Fong Y, Gonen M, Rubin D, Radzyner M, Brennan MF. Long-term survival is superior after resection for cancer in high-volume centers. Ann Surg. 2005;242:540-47. 